\title{
Free videoconferencing apps for the ObGyn
}

\author{
These apps are designed to help clinicians with the transition \\ to a virtual setting
}

Kimia Menhaji, MD, and Katherine T. Chen, MD, MPH
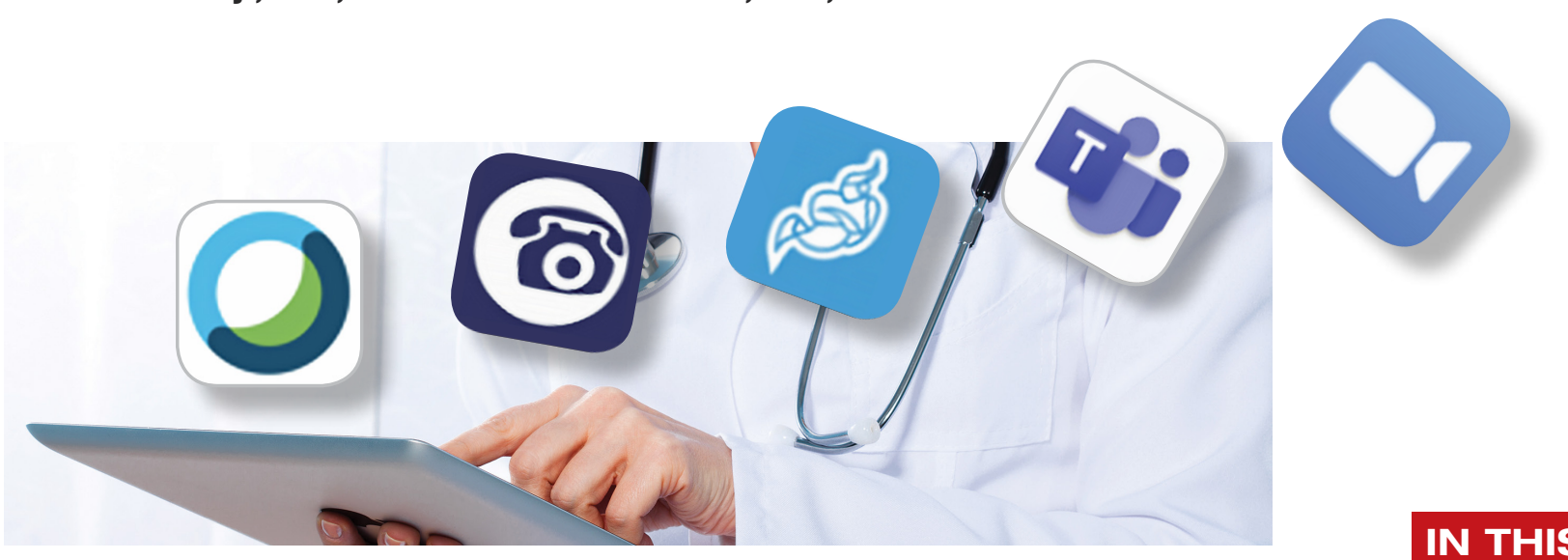

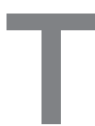
he COVID-19 pandemic has created a metamorphosis in human interactions. One way we have adapted is our increased use of virtual platforms for tasks such as lectures, meetings, interviews, conferences, and patient care via telemedicine. ${ }^{1}$ Virtual platforms have allowed for

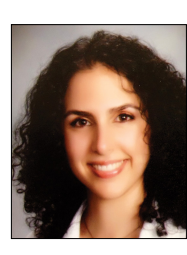

Dr. Menhaji is a third-year Female Pelvic Medicine and Reconstructive Surgery fellow in the Department of Obstetrics, Gynecology, and Reproductive Science, Icahn School of Medicine at Mount Sinai, New York, New York.

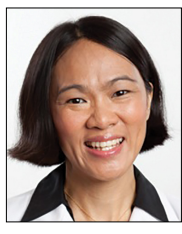

Dr. Chen is Professor of Obstetrics, Gynecology, and Reproductive Science and Medical Education, Vice-Chair of Ob-Gyn Education for the Mount Sinai Health System, Icahn School of Medicine at Mount Sinai, New York. She is an OBG MANAGEMENT Contributing Editor.

Dr. Chen reports being an advisory board member and receiving royalties from UpToDate, Inc. Dr. Menhaji reports no financial relationships relevant to this article. the continuation of existing programs and facilitated new collaborations ranging from international webinars on patient care to national lectures for residents and fellows in ObGyn. New virtual platforms continue to emerge. We present here a review of free virtual communication apps available to the ObGyn care provider.

We used the term "videoconference" to search the Apple and Google Play app stores between May 29, 2020, and June 1, 2020. A total of 25 apps that offered both audio and videoconferencing were identified. All were free for download, but the majority required an ongoing paid subscription fee for the service. Thirteen programs were either completely free or offered a free version of their services. Based on our review and a systematic analysis, we selected 5 apps to feature here: Cisco Webex Meetings, Free Conference Call, Jitsi Meet, Microsoft Teams, and Zoom.

\section{Featured videoconferencing apps}

Cisco Webex Meetings and Free Conference Call offer an easy video meeting setup from 
TABLE Five free videoconference apps and included features

\begin{tabular}{|c|c|c|c|c|c|}
\hline & $\begin{array}{l}\text { Cisco Webex } \\
\text { Meetings }\end{array}$ & $\begin{array}{c}\text { Free } \\
\text { Conference Call }\end{array}$ & Jitsi Meet & $\begin{array}{l}\text { Microsoft } \\
\text { Teams }\end{array}$ & Zoom \\
\hline Devices: iOS and Android & $\checkmark$ & $\checkmark$ & $\checkmark$ & $\checkmark$ & $\checkmark$ \\
\hline Mac, Windows & & & $\checkmark$ & $\checkmark$ & $\checkmark$ \\
\hline Free format & Personal & PWYC & $\mathrm{N} / \mathrm{A}$ & Free & Basic \\
\hline Registration required & $\checkmark$ & $\checkmark$ & $X$ & $\checkmark$ & $\checkmark$ \\
\hline Maximum number of participants & 100 & 1,000 & 75 & $500 \mathrm{~K}$ & 100 \\
\hline Number of meetings & Unlimited & Unlimited & Unlimited & Unlimited & Unlimited \\
\hline Length of $1: 1$ meeting & Unlimited & & & & Unlimited \\
\hline Length of group meeting & $50 \mathrm{~min}$ & & & & $40 \mathrm{~min}$ \\
\hline HD video and/or audio & $\checkmark$ & & & & $\checkmark$ \\
\hline Voice over Internet Protocol & $\checkmark$ & $\checkmark$ & & & \\
\hline Active speaker view & $\checkmark$ & $\checkmark$ & & & $\checkmark$ \\
\hline Full screen and/or gallery view & $\checkmark$ & $\checkmark$ & $\checkmark$ & & $\checkmark$ \\
\hline Screen sharing & $\checkmark$ & $\checkmark$ & $\checkmark$ & $\checkmark$ & $\checkmark$ \\
\hline Multi-share & & $\checkmark$ & & & $\checkmark$ \\
\hline Whiteboard & $\checkmark$ & & & & $\checkmark$ \\
\hline Co-annotation on shared screen & & $\checkmark$ & & & $\checkmark$ \\
\hline File sharing & $\checkmark$ & $\checkmark$ & & $\checkmark$ & \\
\hline Keyboard/mouse control & & & & & $\checkmark$ \\
\hline Virtual background/background blur & & & & $\checkmark$ & $\checkmark$ \\
\hline Instant meetings & $\checkmark$ & & $\checkmark$ & $\checkmark$ & $\checkmark$ \\
\hline Scheduled meetings & $\checkmark$ & $\checkmark$ & & & $\checkmark$ \\
\hline Calendar compatibility & $\checkmark$ & & $\checkmark$ & & $\checkmark$ \\
\hline Chrome and/or Outlook plug-ins & & $\checkmark$ & & & $\checkmark$ \\
\hline Web app-no downloads or plug-ins & $\checkmark$ & & $\checkmark$ & & \\
\hline Custom/unique meeting links & $\checkmark$ & & $\checkmark$ & & $\checkmark$ \\
\hline Polling & $\checkmark$ & & & & \\
\hline Recording & $\checkmark$ & $\checkmark$ & $\checkmark$ & & $\checkmark$ \\
\hline Private chat/messaging & & $\checkmark$ & & & $\checkmark$ \\
\hline Group chat/messaging & $\checkmark$ & $\checkmark$ & $\checkmark$ & $\checkmark$ & $\checkmark$ \\
\hline Host controls & $\checkmark$ & & & & $\checkmark$ \\
\hline Raise hand & $\checkmark$ & & $\checkmark$ & & $\checkmark$ \\
\hline Breakout rooms & & $\checkmark$ & & & $\checkmark$ \\
\hline Secure socket layer (SSL) encryption & & & & & $\checkmark$ \\
\hline Personal room locking and unlocking & $\checkmark$ & $\checkmark$ & $\checkmark$ & & \\
\hline Additional features & & $\begin{array}{c}\text { Integrate with } \\
\text { other apps: } \\
\text { Slack, } \\
\text { Evernote, } \\
\text { Dropbox }\end{array}$ & $\begin{array}{l}\text { Available } \\
\text { for Slack }\end{array}$ & $\begin{array}{l}\text { Real time } \\
\text { collaboration: } \\
\text { Word, Excel, } \\
\text { PowerPoint, } \\
\text { and OneNote }\end{array}$ & $\begin{array}{l}\text { Closed } \\
\text { captioning }\end{array}$ \\
\hline
\end{tabular}

Abbreviations: min, minutes; N/A, not applicable; PWYC, pay what you can; U, unlimited. 
both a smartphone and a desktop app. They provide seamless access to functions on the virtual main page, including chat with other participants in the meeting and screen sharing. These apps both require screen recording in order to share screens.

Jitsi Meet is a web app usable on an iPhone or Android as well as on a desktop through the meet.jit.si website. No account is required. On the app or website, the user creates a meeting name and shares the unique URL or meeting name with invitees to join the videoconference. The mobile app and website both offer a "raise your hand" feature, full screen and/or gallery (tile) view, group chat, and live streaming. In both settings, users may lock the meeting and require a password. Additional features through the website include screen sharing, recording the meeting, blurred background, muting all participants, and sharing YouTube videos.

The Microsoft Teams app asks you the purpose of signing up on the website- "use for school," "with friends and family," or "for work." If you choose "with friends and family," the app directs you to Skype. Choosing the "for work" function directs you to complete your free registration. Microsoft Teams requires participants to create teams; thus, others participating in the videoconference need to have their own account. However, "guest access" also is available.

On the Zoom platform, immediate and scheduled meetings can be set up on the app as well as on the website, or directly on Microsoft Outlook and Google Calendar if the plug-in has been established. The desktop and smartphone apps are similar in function and provide access to personalized settings.

For patient care, since HIPAA (Health Insurance Portability and Accountability Act) protection is a concern, we recommend following guidelines at the user's institution regarding use of apps such as Epic Haiku for telehealth visits. For teaching and interacting with colleagues, we recommend Cisco Webex, Free Conference Call, Microsoft Teams, and Zoom, keeping in mind the time limitations of each app for the free account.

Overall, these 5 apps are easy to set up and user-friendly. Deciding which program to choose will depend on the number of participants allowed for a meeting and the duration of the meeting, as these two factors seem to be the most constraining among the free videoconferencing apps.

References

1. Karram M, Baum N. Telemedicine: a primer for today's ObGyn. OBG Manag. 2020;32(5):28-32. 